\title{
Measurement of the phase and group refractive indices, and the dispersion of the thermo-optic and strain-optic coeffients of optical fibers using weak fiber Bragg gratings
}

\author{
J. L. Cruz, ${ }^{1, *}$ Y. O. Barmenkov, ${ }^{2}$ A. Díez, ${ }^{1}$ And M.V. Andres ${ }^{1}$ \\ ${ }^{1}$ Department of Applied Physics (ICMUV), University of Valencia, Dr. Moliner 50, Burjassot 46100, Spain. \\ ${ }^{2}$ Centro de Investigaciones en Óptica A.C., León 37150, México. \\ *Corresponding author: cruz@uv.es
}

Received XX Month XXXX; revised XX Month, XXXX; accepted XX Month XXXX; posted XX Month XXXX (Doc. ID XXXXX); published XX Month XXXX

In this work we report on the measurement, with record accuracy, of the absolute modal effective refraction index (phase index) of single mode optical fibers by using Bragg gratings. We also demonstrate a new method to measure the group index of the fibers from the gratings Bragg wavelength. We present as well the characterization of the thermo-optic and strain-optic coefficients as a function of the wavelength, the values we have obtained are the closest to those of the pristine fiber measured with gratings technology so far. The phase index is measured with a set of gratings in the wavelength range from $1509 \mathrm{~nm}$ to $1563 \mathrm{~nm}$ and the group index is obtained from the wavelength dependence of the phase index. Very weak gratings with reflectivity down to $10^{-3}$ have been used in order to minimize the perturbation of the pristine fiber. Results are presented at a temperature of $22^{\circ} \mathrm{C}$ and zero strain after preliminary calibration of the thermo-optic and strain-optic coefficients as a function of the wavelength. Accuracies better than $3 \times 10^{-5}$ and $7 \times 10^{-4}$ have been achieved for the phase and group indices, respectively. It is also shown that the main source of error relates to the uncertainty in pitch of the phase masks used for gratings inscription. The technique is useful for testing different kinds of fibers including telecommunication, amplifying, and polarization maintaining. (C) 2020 Optical Society of America

OCIS codes: 060.2310 Fiber optics; 060.2400 Fiber properties; 060.3735 Fiber Bragg gratings; 120.4530 Optical constants.

\section{INTRODUCTION}

Phase and group refractive indices of the fundamental mode in optical fibers are key parameters for accurate design and characterization of optical systems in such fields as telecommunications, laser technology, nonlinear phenomena, metrology or sensors. The two indices can be calculated from measurements of the index profile in the cross section of the fibers [1] or of fiber the preforms [2]. These interferometric or diffractive techniques require special calculation software since actual fibers do not have an exact a step index profile and, what is more, the index profile is measured with reference to a matching index liquid [3, 4], which imposes a limit to the accuracy of results. For example, Cargille Laboratories provides fused silica matching fluids calibrated in wavelength and temperature with an accuracy of $1 \times 10^{-4}$ [5]. An index resolution in fiber of $1 \times 10^{-4}$ is achieved in [3] and a discrepancy between measurement methods of $5 \times 10^{-4}$ has been observed in [4], these errors propagate in the subsequent calculation of the phase and group indices.

Alternatively, the group index can be directly measured in the fibers by pulse propagation or by phase modulation method [6] and, additionally, the refractive index of bulk materials $[7,8]$ and the group index of fibers $[9,10]$ have been measured by interferometric setups. These techniques are based on the phase or on the group time cumulated over a given length of fiber. Interferometric techniques can in principle provide accuracies of $10^{-5}$ or better when the phase index is measured in bulk media, however the length of the samples under study, among other requirements, must be known with high precision. For example, the index of a standard referenced material has been measured by an interferometric technique in reference [8], the authors obtain an accuracy of $1.1 \times 10^{-5}$ after measuring a thickness of 856.40 $\mu \mathrm{m}$ with an accuracy of $0.01 \mu \mathrm{m}$. However, in references $[9,10]$ the group index of optical fibers is measured by interferometry with an accuracy of $7 \times 10^{-4}$, the fiber length precision being a limiting factor. Although physical lengths can be measured with extremely high precision in air [11], only the optical path length can be measured with 
extreme precision in optical fibers [12], and the measurement of an absolute fiber length with a relative accuracy better than $10^{-4}$ at a controlled temperature and strain is not an attainable task in many cases. The measurement of modal phase indices in few modes or multimode fibers relies on the previous knowledge of the fundamental mode $[13,14,15]$ since the measurement techniques only provide information on the index difference between modes. Fiber birefringence depends on the difference of two orthogonal refraction indices and can be measured with very high precision by interferometric or by OTDR techniques $[16,17]$. In summary, the determination the absolute value of the phase index in fibers with an accuracy below $10^{-4}$ is still a challenging task because the accuracy of interferometric techniques is limited by the fiber length accuracy (when measuring effective index directly) or by the accuracy of the matching index fluids (when measuring the index profile in the fiber cross section). Therefore, alternative techniques are required to provide solutions to a vast community.

Recently, Jülich and Roths reported on the determination of the phase index from the Bragg wavelength of a fiber grating and demonstrated an accuracy of $1.4 \times 10^{-4}$ at a wavelength of $\sim 1535 \mathrm{~nm}$ [18]. Following Jülich's proposal, we report in this work the measurement of the absolute value of the phase index using gratings of very low reflectivity $\left(R \sim 10^{-3}\right)$. These gratings do not significantly perturb the refraction index of the pristine fiber. We reduce the incertitude of experimental data to a level close to the limit imposed by the accuracy of the pitch of the phase masks used for gratings inscription, which allows one to measure the wavelength dependence of the phase index with higher accuracy and, therefore, the calculation of the group index. The phase index was measured at twelve different wavelengths with a set of gratings in the band from 1509 to $1563 \mathrm{~nm}$. Experimental data has a standard error below $3 \times 10^{-5}$ and the group index was determined from the phase index with an accuracy better than $7 \times 10^{-4}$. The temperature and strain of the fibers when the gratings were fabricated were considered to achieve the desired accuracy, and hence the strain-optic and thermo-optic coefficients were measured in the spectral range. These coefficients have been measured because there is a discrepancy of values in the literature. Because of the low reflectivity of the gratings, the values of these coefficients are the closest to those of the pristine fiber reported so far using fiber gratings.

The experimental results are presented for three kinds of fibers: telecommunication, erbium doped and polarization maintaining ones.

The novelties of this work can be summarized as follows: we have measured phase index of optical fibers with record precision, we have demonstrated a new method to measure the group index using Bragg gratings, we have measured wavelength dependence of the thermooptic and strain-optic coefficients and our values are the closest to those of the pristine fiber reported so far.

\section{CONCEPTS AND METHODS}

A fiber Bragg grating consists of a periodic modulation of the refractive index of the optical fiber that couples counter-propagating modes. The $\mathrm{HE}_{11}$ core mode in a single mode fiber is reflected at the wavelengths that accomplish the Bragg condition. The fundamental resonance appears at the Bragg wavelength $\lambda_{B}$ given by the effective phase index of the propagating mode $n_{\text {eff }}(\lambda)$ and the grating period $\Lambda$. If the grating is written using a phase mask of period (pitch) $D$, then the grating period is a half of the mask pitch [19]:

$$
\lambda_{B}=2 n_{\text {eff }}(\lambda) \Lambda=D n_{\text {eff }}(\lambda) .
$$

The measurement of $\lambda_{B}$ in gratings with different periods allows one to measure the wavelength dependence of the phase index and, consequently, the group index can be obtained from the expression:

$$
n_{g}=n_{\text {eff }}(\lambda)-\lambda \frac{d n_{\text {eff }}(\lambda)}{d \lambda} \text {. }
$$

When a grating is fabricated at a temperature $T$ the phase mask has pitch $D(T)$ that is different from the nominal value $D\left(T_{\text {ref }}\right)$ provided by the mask manufacturer and correspondent to the reference temperature $T_{\text {ref }}=22{ }^{\circ} \mathrm{C}$. Gratings are usually fabricated under some strain $\varepsilon$, which is applied in order to keep the fiber straight to prevent chirp. The grating spectra are measured just after the grating inscription, therefore the measured Bragg wavelength depends on both temperature $T$ and strain $\varepsilon$. In such conditions, the value of the phase refractive index $n_{e f f}(\lambda, T, \varepsilon)$ is related to the measured Bragg wavelength $\lambda_{B}(T, \varepsilon)$ as:

$$
n_{\text {eff }}(\lambda, T, \varepsilon)=\frac{\lambda_{B}(T, \varepsilon)}{D_{(T)}} .
$$

The phase index must be converted to the reference temperature and to zero strain for standardization. The conversion also compensates the temperature variations during the fabrication process. A linear approximation of equation (3) gives the value of the index at zero strain and $T_{\text {ref }}$ as a function of the measured Bragg wavelength $\lambda_{B}(T, \varepsilon)$ and the nominal value of the mask pitch $D($ Tref):

$$
n_{e f f}(\lambda, \text { Tref }, \varepsilon=0)=\frac{\lambda_{B}(T, \varepsilon)}{D_{(\text {Tref })}}\left[1+p_{e} \varepsilon-\left(\zeta+\alpha_{m}\right)\left(T-T_{\text {ref }}\right)\right],
$$

where $\alpha_{m}$ is the thermal expansion coefficient of the mask, $\zeta$ and $p_{e}$ are the thermo-optic and the strain-optic coefficients of the fiber:

$$
\begin{aligned}
& \zeta=\frac{1}{n_{\text {eff }(\lambda)}} \frac{\partial n_{\text {eff }(\lambda)}}{\partial T}, \\
& p_{e}=\frac{1}{n_{\text {eff }(\lambda)}} \frac{\partial n_{\text {eff }(\lambda)}}{\partial \mathcal{E}} .
\end{aligned}
$$

The phase index of a step index fiber has a theoretical variation with wavelength $d n_{\text {eff }} / d \lambda$ of the order of $-10^{-5} \mathrm{~nm}^{-1}$, therefore it is expected an experimental variation of the phase index of the order of $10^{-4} \mathrm{~nm}$ in a span of $10 \mathrm{~nm}$. As a consequence, if we want to obtain a reliable value of the group index, we need to measure the phase index with an accuracy of the order of $10^{-5}$ in an interval of few tens of $\mathrm{nm}$. This accuracy can be achieved only when a number of experimental parameters are well controlled, these parameters involve fabrication errors, measurement errors and environmental errors. We discuss below these errors which are summarized in Table 1.

\section{Fabrication errors:}

When a grating is fabricated by laser exposure (UV light in our case) the phase index of the pristine fiber $n_{e f f}(\lambda)$ is modified and, as a consequence, the Bragg wavelength shifts to a value $\lambda_{\max }$ that differs from the value $\lambda_{B}$ expected from $n_{\text {eff }}(\lambda)$ and $D ; \lambda_{\max }$ can be written in the form:

$$
\lambda_{\max }=\lambda_{B}\left(1+\frac{\Delta \bar{n}}{n_{e f f}(\lambda)} f_{p c}\right)^{\prime}
$$

where $\Delta \bar{n}$ is the mean perturbation of the index produced by the UV exposure, and $f_{p c}$ is the fraction of power of the HE11 mode confined in the photosensitive fiber core (confinement factor). The index perturbation can be over $10^{-4}$ in strong gratings [20]. Such perturbation is excessive for an accurate determination of the phase index because we require an index accuracy of $10^{-5}$; we have prevented this problem by using gratings of small index modulation (low reflectivity gratings). The diffraction efficiency of commercial phase masks is high and the diffraction order 0 carries less than $3 \%$ of the power carried by the \pm 1 diffraction orders, therefore $\Delta \bar{n}$ is approximately equal to the amplitude $\Delta n$ of the sinusoidal index 
modulation. The amplitude of the index modulation determines in turn the maximum power reflectivity $R$ as:

$$
R=\tanh ^{2}\left(\frac{\pi L}{\lambda} \Delta n f_{p c}\right)=\tanh ^{2}\left(\frac{\pi L V}{\lambda_{c} V_{c}} \Delta n f_{p c}\right),
$$

where $L$ is the physical grating length, $\lambda_{c}$ the fiber cut-off wavelength, $V$ the normalized frequency and $V_{c}$ the normalized cut-off frequency $\left(V_{c} \approx 2.405\right)$. According to equation (8), a low reflectivity $R$ requires a small index modulation $\Delta n$, the index in the grating at low $\Delta n$ approaches to the index of the pristine fiber; as a consequence, the measured peak wavelength $\lambda_{\max }$ is close to $\lambda_{B}$ according to (7) and the index of the pristine fiber can be calculated using equation (4).

The index perturbation has been calculated using equation (8) for gratings of different reflections and the result is represented in Fig. 1 as a function of the normalized frequency; the confinement factor $f_{p c}$ has been calculated using Marcuse's formulae [21]. The simulation corresponds to a grating length $L=1 \mathrm{~cm}$ and a cut-off wavelength of $\lambda_{c}=$ $1 \mu \mathrm{m}$, it is straight forward to scale the results for other values of $L$ or $\lambda_{c}$ through the product $\Delta n L / \lambda_{c}$. It can be seen in Fig. 1 that gratings with reflectivity $R \sim 10^{-3}$ experience an index perturbation $\Delta n \sim 2 \times 10^{-6}$. The relative variation of the reflected wavelength $\left(\lambda_{\max }-\lambda_{B}\right) / \lambda_{B}$ has been calculated for these gratings using equation (7), the relative variation is of the order of $10^{-6}$ when the reflectivity is about $10^{-3}$; this relative variation corresponds to absolute wavelength variations below $2 \mathrm{pm}$ for a Bragg wavelength around $1550 \mathrm{~nm}$. Thus, we can conclude that the Bragg condition of equation (1) predicts the phase refractive index with an accuracy of $10^{-6}$ if the grating reflectivity is approximately $10^{-3}$.
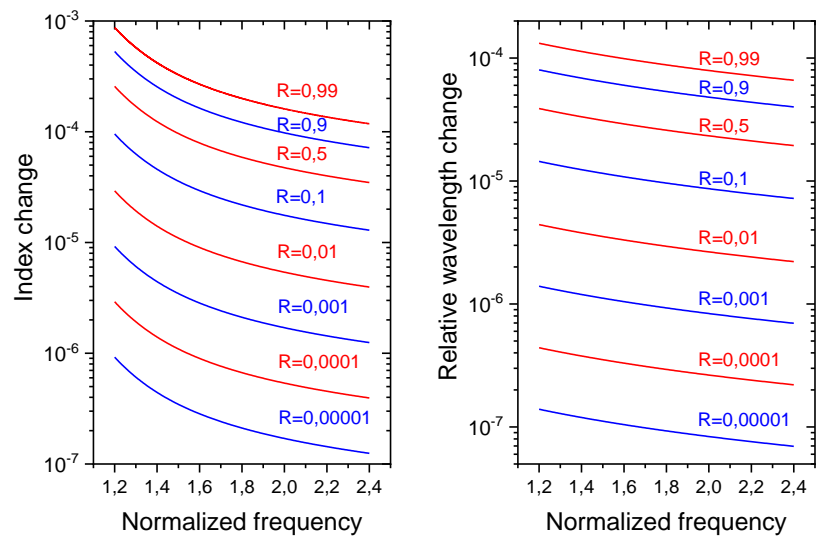

Fig. 1. Left: perturbation of the refractive index as a function of the normalized frequency in gratings of different reflections. Right: relative variation of the reflected wavelength.

To verify this idea a grating was fabricated by scanning several times a sample of Corning-SMF28 fiber with a UV beam through a phase mask. The gratings were $1 \mathrm{~cm}$ in length. The grating spectra where recorded after each irradiation and the spectra corresponding to each superimposed scan are plotted in Fig. 2. It can be observed that the main reflection lobe rises at constant wavelength. The grating reflectivity can be inferred from the reflection of the fiber tip which was terminated at right angle before the gratings inscription in order to have a reference for a reflection level of about 0.033 . The grating spectra have a reflectivity ranging between 0.013 and 0.0013 , the difference of the Bragg wavelengths of the five spectra is below $4 \mathrm{pm}$ (close to the repeatability of wavelength measurements), this small difference agrees with the theoretical prediction.

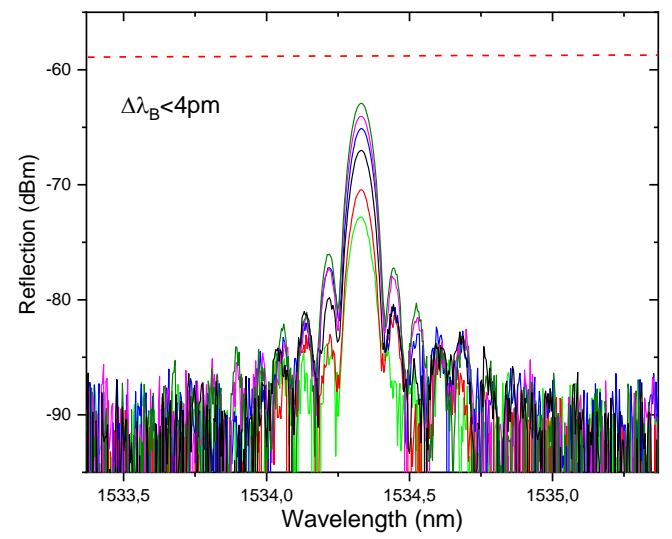

Fig. 2. Grating spectra as the reflectivity rises from 0.0013 to 0.013 . Each spectrum from bottom to top corresponds to one superimposed writing scan. The dashed top line indicates a reference reflection level of 0.033 . The peak wavelength shifts to the red about $4 \mathrm{pm}$.

A second source of incertitude is the pitch of the phase mask, phase masks manufactured by Ibsen Photonics have a period accuracy of $\pm 10 \mathrm{pm}$, therefore the incertitude in the phase index is about $\pm 1.35 \times 10$ 5 for a mask of pitch $D=1070 \mathrm{~nm}$ producing gratings at $1550 \mathrm{~nm}$. The phase mask error is the main contribution to the overall error in the experiments as it can be seen in Table 1 .

The tilt angle between the mask and the fiber is measured with an accuracy of $9.5 \times 10^{-4}$ rad., which modifies the pitch with respect to the fiber and adds an error to the phase index of $\pm 8 \times 10^{-7}$. The incidence angle of the UV laser on the phase mask is measured with an accuracy of $6.7 \times 10^{-3} \mathrm{rad}$. and produces an index error of $\pm 4 \times 10^{-7}$. The total contribution of misalignments to the index error is $\pm 9 \times 10^{-7}$.

Finally, actual fibers are not perfectly uniform along the fiber axis, it has been demonstrated that short pieces of fiber may exhibit index irregularities that affect both core and cladding modes [15, 22, 23]. The index variations along the fiber can range between $\pm 2 \times 10^{-6}$ for a SMF28 fiber to $\pm 1 \times 10^{-5}$ for polarization maintaining or high numerical aperture fibers [23].

Table 1. Phase index uncertainty due to different parameters.

\begin{tabular}{llllllll}
\hline UV induced & Phase mask & Angles & $\begin{array}{l}\text { Fiber } \\
\text { uniformity }\end{array}$ & Temperature & Zero strain & $\begin{array}{l}\text { Wavelength } \\
\text { measurement }\end{array}$ \\
\hline $0.2 \times 10^{-5}$ & $1.35 \times 10^{-5}$ & $0.09 \times 10^{-5}$ & $(0.2$ to 1$) \times 10^{-5}$ & $0.43 \times 10^{-5}$ & $0.06 \times 10^{-5}$ & $0.33 \times 10^{-5}$ & $\pm\left(\mathbf{1 . 6}\right.$ to 1.9) $\times \mathbf{1 0}^{-5}$ \\
\hline
\end{tabular}




\section{Environmental errors:}

The index depends also on temperature and strain according to the thermo-optic and the strain-optic coefficients $\zeta$ and $p_{e}$. To obtain the refractive index with more than three significant digits the temperature and strain of the fiber must be specified. Temperature has been measured with an accuracy $0.5{ }^{\circ} \mathrm{C}$; thus, from the measurements of $\zeta$ reported in section 2, this thermal error results in an index incertitude of $\pm 4.3 \times 10^{-6}$. Fibers are always subjected to a residual strain, either under compression, expansion or torsion. The strain uncertainty was determined by removing several times the load that keeps the fiber straight in the fabrication rig and measuring the Bragg wavelength of the unloaded grating. The measured data present some dispersion due to the bend of the unloaded fiber and to the residual friction of the fiber on the holders. An index uncertainty of $6 \times 10^{-7}$ was estimated from the dispersion of the Bragg wavelength and the strainoptic coefficients presented in section 2 . The actual error could be even lower than the estimated value because the wavelength shift was only slightly higher than the repeatability of the spectrum analyzer. Note that the variation of the atmospheric pressure has negligible effect.

Apart from the error in measuring temperature and strain, the fabrication process involves larger variations of the room temperature that must be measured and compensated. In a similar way, the gratings have to be written under some not negligible strain that must be measured and corrected for an accurate determination of $n_{e f f}$.

\section{Measurement errors:}

The gratings spectra are measured with an optical spectrum analyzer (OSA Yokogawa model AQ6370-C) that has a specified accuracy of $10 \mathrm{pm}$. The actual accuracy of our instrument was obtained by a simultaneous measurement of a laser line with the OSA and with a wavelength meter (Burleigh WA-1650) operating by interferometry with respect to an in-built wavelength standard, the operation principle can be found in [6]. The wavelength meter has an accuracy of $0.3 \mathrm{pm}$. The OSA was calibrated with its internal light source before the accuracy test. The test was performed at twelve discrete wavelengths between 1510 to $1565 \mathrm{~nm}$, the spectral range was scanned nineteen times in a lapse of time of three hours, the temperature in the laboratory rose two degrees during the experiment.

The difference of the readings of both instruments is plotted in Fig. 3. It is seen that the experimental data are randomly distributed in time and in wavelength. The inaccuracy has a slow tendency to rise in the lapse of three hours which is conditioned by the increment of temperature during the test. About $93 \%$ of data points are measured by the OSA with an accuracy better than $3.5 \mathrm{pm}$. The uncertainty in the wavelength results in a fiber index inaccuracy of $\pm 3.3 \times 10^{-6}$.

From the combination of all the errors mentioned above, we estimate that the absolute value of the phase index of the $\mathrm{HE}_{11}$ propagation mode can be measured with an accuracy of $\pm 1.8 \times 10^{-5}$, the phase mask error being the main contribution to the overall error.

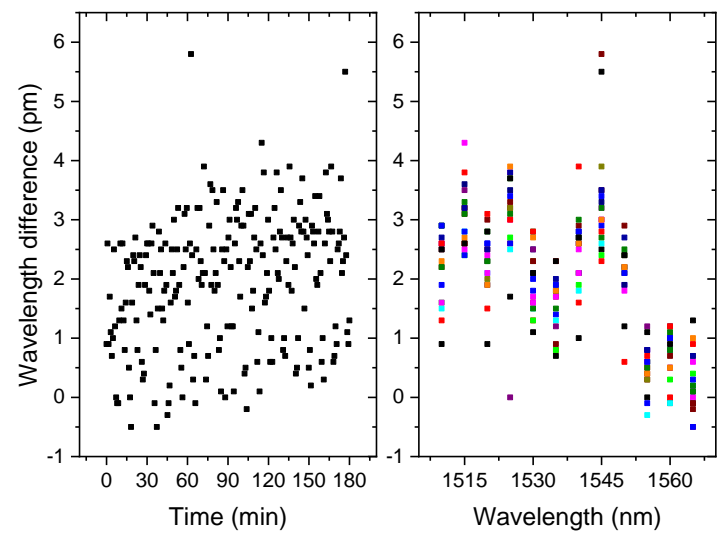

Fig. 3. Absolute accuracy of the OSA: difference between the wavelengths measured by the OSA and by the interferometric wavelength meter. Left: data as a function of time. Right: data as a function of wavelength.

\section{EXPERIMENT AND RESULTS}

Three fibers have been studied in this work: the Corning SMF 28e+ telecommunication fiber, the Fibercore M5(980/125) erbium doped fiber and the Fibercore $H B 1250$ polarization maintaining fiber. The fibers have numerical apertures of $0.14,0.23$ and 0.15 , and cut-off wavelengths of 1260,939 and $1180 \mathrm{~nm}$ respectively. The $H B 1250$ fiber has a beat length of $1.6 \mathrm{~mm}$.

Twelve gratings haven been inscribed in each fiber using phase masks provided by Ibsen Photonics. Ten of the mask have their pitch equally spaced between $1042.50 \mathrm{~nm}$ and $1074.00 \mathrm{~nm}$, the two remaining masks have periods of 1076.25 and $1079.05 \mathrm{~nm}$. The masks have $10 \mathrm{~mm}$ in length. Gratings were fabricated by scanning the fibers through the phase mask with an UV beam at $244 \mathrm{~nm}$ generated by a doubled argon laser. The fibers were not hydrogenated and the laser beam was not focused. We have fabricated gratings with a reflectivity around $10^{-3}$ for two reasons: first, this reflectivity is low enough to produce an index error smaller than other dominant factors, as it is shown in Table 1; second, the reflectivity is high enough to achieve a good signal level in the spectrum analyzer, as it can be seen in Fig. 4 and Fig. 5. The signal measured with a resolution of $17 \mathrm{pm}$ is between 75 and $-80 \mathrm{dBm}$ in our measurement setup and is $20 \mathrm{~dB}$ over the noise level of the spectrum analyzer.

The gratings reflection spectra are shown in Fig. 4. A superluminescent diode centered at $1550 \mathrm{~nm}$ was used as light source. The 3.3\% reflection level from the fiber termination was measured and recorded prior to the grating fabrication in order to guarantee the low reflectivity of the gratings. By comparison with the reference level it can be observed that the gratings reflectivity is less than $0.3 \%$ in all cases (the gratings peaks and the reference level differ more than 10 $\mathrm{dB}$ ). The fluorescence of the erbium doped fiber M5 can be observed in its spectrum. The gratings bandwidth is about $75 \mathrm{pm}$ measured at $3 \mathrm{~dB}$ from the peak, the central wavelengths of the reflection peaks were measured by a parabolic fit of the spectra it as shown in the upper plot of figure Fig. 5. The spectral profile of the superluminescent diode has maximum slope of $0.25 \mathrm{~dB} / \mathrm{nm}$ at $1510 \mathrm{~nm}$, therefore a maximum variation of $0.02 \mathrm{~dB}$ within the grating bandwidth, the convolution of this smooth variation with the grating spectrum does not affect the determination of the central wavelength. 
Each grating written in the polarization maintaining fiber has two reflection bands corresponding to two orthogonal polarization states, the reflectivity level of both states is basically the same because the light source is randomly polarized. It has been tested whether the gratings in the SMF and the M5 fibers had polarization dependence, the test was performed by polarizing the diode light with an external fiber polarizer and using a polarization state controller. Polarization dependency was only observed in the HB fiber as it is shown in Fig. 5 (bottom plot), the spectral variations observed in the other two fibers when the polarization state was scanned are within the repeatability of the spectrum analyzer.

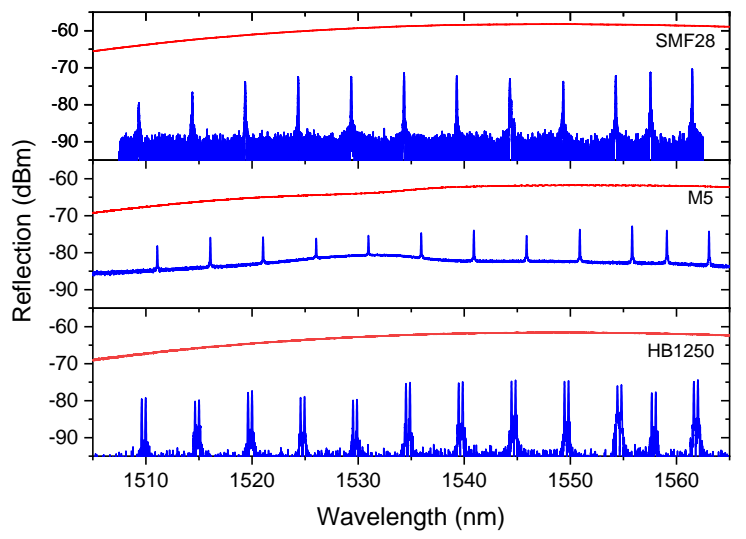

Fig. 4. Spectra of the sets of gratings in the three kinds of fibers. The red line corresponds to the reflection of the right angle terminated fiber tip.
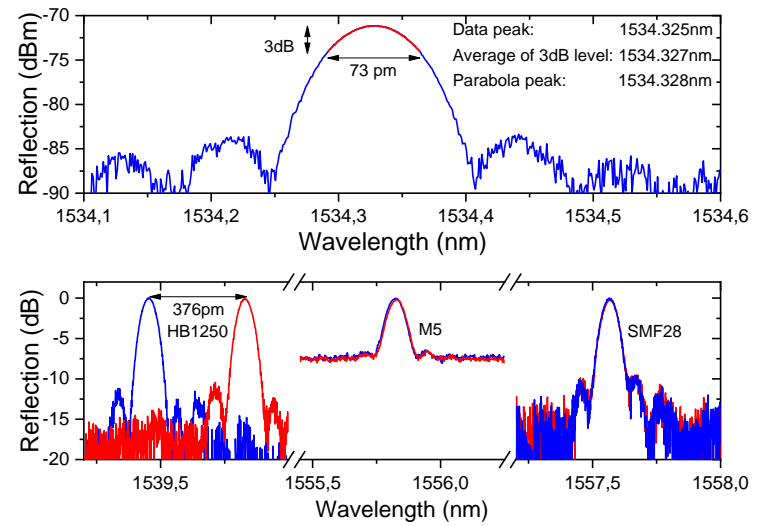

Fig. 5. Top: parabolic fit for Bragg wavelength determination. Bottom: polarization dependence of the reflected spectra in the three kinds of fiber (blue lines: fast axis, red lines: slow axis).

Each array of 12 gratings was fabricated in a single piece of fiber, the total length of each fiber was about $18 \mathrm{~cm}$. All the gratings were recorded under a controlled strain of $\varepsilon=4.77 \times 10^{-5}$ in order to keep the fibers straight and avoid chirp. The strain was applied by loading the fibers with a mass of $4.320 \pm 0.003 \mathrm{~g}$ under the action gravity, the strain is calculated from the load using a Young modulus of silica of $(72.3+772.4 \varepsilon) \times 10^{9} \mathrm{~N} / \mathrm{m}^{2}[24,25]$, a nominal fiber diameter of $125.0 \pm 0.7 \mu \mathrm{m}$ and a value of gravity in our laboratory of $9.8014 \mathrm{~m} / \mathrm{s}^{2}$ located at the see level in latitude $39.4698^{\circ} \mathrm{N}$ [26]. The mass was measured with a balance that has an accuracy of $1 \mathrm{mg}$, the error of 3 $\mathrm{mg}$ corresponds to the mass of the hanging piece of fiber where the load was attached. The effect of this strain must be compensated in order to report the effective index at zero strain. We measured the temperature at which each individual grating was fabricated, the spectrum of each single grating was recorded after the grating was written. The temperature in fabrication rig varies less than $1^{\circ} \mathrm{C}$ during the fabrication of one array of gratings, and it can change in about $\pm 1^{\circ} \mathrm{C}$ from one day to another, the variations of temperature were measured with a resolution of $0.01^{\circ} \mathrm{C}$. The effect of these thermal variations must be compensated for a precise determination of the phase index.

The effective index is obtained from the measured Bragg wavelength and the nominal pitch of the phase mask, it is presented at zero strain and $T_{r e f}=22{ }^{\circ} \mathrm{C}$ using equation 4 . The correction depends on the strainoptic and thermo-optic coefficients of the fibers, these coefficients are dependent on the fiber dopants $[27,28]$ and on the wavelength [29, 30]. The accurate determination of the fiber index requires a measurement of these two coefficients for the specific fibers and operation wavelengths. The Bragg wavelength shift was measured when the gratings were subjected to variations of temperature or strain and the coefficients were obtained from equations:

$$
\begin{aligned}
& \frac{\Delta \lambda_{B}}{\lambda_{B}}=\left(\alpha_{f}+\zeta\right) \Delta T, \\
& \frac{\Delta \lambda_{B}}{\lambda_{B}}=\left(1-p_{e}\right) \Delta \varepsilon,
\end{aligned}
$$

where $\alpha_{f}$ is the thermal expansion coefficient of the fiber which can be approximated by the one of silica glass $\left(\alpha_{y}=\alpha_{m}=5.1 \times 10^{-7} \mathrm{~K}^{-1}\right.$ at $22^{\circ} \mathrm{C}$ [24]).

A climatic chamber was used for the thermal test at seven discrete temperatures between 5 and $35{ }^{\circ} \mathrm{C}$, the temperature stability was better than $0.5{ }^{\circ} \mathrm{C}$. The gratings were subjected to a thermal cycle before the main test. The three sets of gratings were tested and a linear variation of the Bragg wavelength with temperature was obtained in all cases, we did not observe nonlinear behavior in this short temperature range $[31,32]$. The experimental data were fitted by equation (9) to obtain the thermo-optic coefficients of the fibers, all the thirty-six linear fits had a correlation coefficient R-squared higher than 0.9998 . The thermo-optic coefficients as a function of the wavelength are represented in Fig. 6.
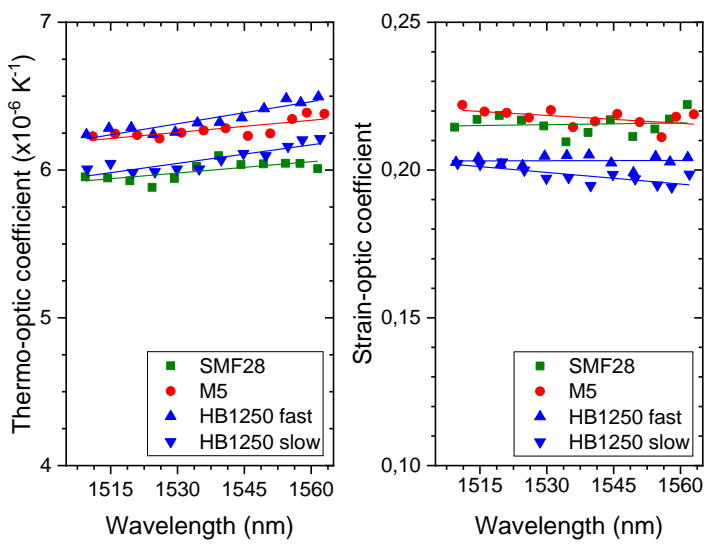

Fig. 6. Thermo-optic (left) and strain-optic (right) coefficients of the fibers as a function of the wavelength. 
Table 2. Linear fit of the thermo-optic $(\zeta)$ and the strain-optic $\left(p_{e}\right)$ coefficients as a function of the wavelength.

\begin{tabular}{lccccccc}
\hline & \multicolumn{3}{c}{$\zeta=A+B(\lambda-1535)$} & & \multicolumn{3}{c}{$p_{e}=A+B(\lambda-1535)$} \\
\cline { 2 - 3 } \cline { 6 - 8 } & $\mathrm{A}\left(\mathrm{K}^{-1}\right)$ & $\mathrm{B}\left(\mathrm{K}^{-1} \mathrm{~nm}^{-1}\right)$ & Standard error & & $\mathrm{A}$ & $\mathrm{B}\left(\mathrm{nm}^{-1}\right)$ & Standard error \\
\hline SMF28 & $5,99 \times 10^{-6}$ & $2.6 \times 10^{-9}$ & $\pm 0.05 \times 10^{-6}$ & & 0.215 & $2 \times 10^{-5}$ & \pm 0.004 \\
M5 & $6.27 \times 10^{-6}$ & $2.7 \times 10^{-9}$ & $\pm 0.04 \times 10^{-6}$ & & 0.218 & $-9 \times 10^{-5}$ & \pm 0.003 \\
HB1250 fast & $6.34 \times 10^{-6}$ & $5.0 \times 10^{-9}$ & $\pm 0.04 \times 10^{-6}$ & & 0.203 & $0.3 \times 10^{-5}$ & \pm 0.003 \\
HB1250 slow & $6.07 \times 10^{-6}$ & $4.2 \times 10^{-9}$ & $\pm 0.04 \times 10^{-6}$ & & 0.199 & $-13 \times 10^{-5}$ & \pm 0.003 \\
\hline
\end{tabular}

The coefficients at $1535 \mathrm{~nm}$ are comprised between $5.99 \times 10^{-6} \mathrm{~K}^{-1}$ for the SMF-28 fiber and $6.34 \times 10^{-6} \mathrm{~K}^{-1}$ for the fast axis of the birefringent fiber, the coefficients have a small variation with the wavelength comprised between $2.6 \times 10^{-9} \mathrm{~K}^{-1} \mathrm{~nm}^{-1}$ and $5.0 \times 10^{-9} \mathrm{~K}^{-1} \mathrm{~nm}^{-1}$ for the same fibers. The linear fits of the thermo-optic coefficients are presented in Table 2, the fitting lines predict the wavelength dependence of the coefficient with a standard error of about $0.04 \times 10^{-6}$, the error is obtained from the residual sum of squares. It is worth observing that the two axes of the birefringent fiber present different thermal response: the coefficient of the fast axis is about $4 \%$ higher than the slow one. Our results are in agreement with the generic value of $\zeta=6,16 \times 10^{-6} \mathrm{~K}^{-1}$ given in reference [33] which corresponds to a temperature sensitivity $d \lambda_{B} / d T=10.3 \mathrm{pm} / \mathrm{K}$ at $1548.7 \mathrm{~nm}$, however it must be noticed that values higher and lower than ours have been reported for the SMF28 fiber: $5.32 \times 10^{-6} \mathrm{~K}^{-1}$ at $1548.7 \mathrm{~nm}$ in [32] and $(7.5 \pm 0.3) \times 10^{-6} \mathrm{~K}^{-1} 1544.2 \mathrm{~nm}$ in [34]. Our estimation from the fitting equation of Table 2 is $\zeta=(6.03 \pm 0.05) \times 10^{-6}$ at $1548.7 \mathrm{~nm}$, the corresponding thermal sensitivity of is $10.1 \mathrm{pm} / \mathrm{K}$. The differences may be due to the type and the strength of the gratings written in each case that can modify the response of the pristine fiber [35].

The strain-optic coefficients were obtained by loading the fibers with seven calibrated masses up to a maximum load of $26.884 \mathrm{~g}$, the uncertainty of the load applied to each set of gratings was the weight of the hanging piece fiber $(0.020 \mathrm{~g})$, the nonlinear change of Young modulus of silica is less than $0.3 \%$ in this range of load [25]. The response was measured and fitted with equation (10), the linear fits corresponding to each grating had a correlation coefficient higher than 0.99995, the slope of each line provides the coefficient at the specific wavelength. The strain-optic coefficients as a function of the wavelength are plotted in Fig. 6 and the fitting lines of the coefficients are given in Table 2. The coefficients at $1535 \mathrm{~nm}$ are comprised between 0.199 for the slow axis of the HB1250 fiber and 0.218 for the M5 fiber, wavelength variations are only observed in the erbium doped fiber M5 and in the fast axis of the birefringent fiber, both fibers present coefficients with negative slopes. The coefficients of the HB1250 fiber are smaller than the other two fibers, furthermore the two axes of this fiber have the same coefficient at $1504.9 \mathrm{~nm}$ but the slopes of the two axes are clearly different, this is a consequence of the cladding structure of this fiber. The fitting parameters of Table 2 allows one to predict the wavelength dependence of the coefficients with a standard error of about 0.003 , the major contribution to this error is the uncertainty in the fiber diameter $( \pm 0.7 \mu \mathrm{m})$. Reference [33] gives a commonly accepted value for silica fibers of $p_{e}=0.22$ (strain sensitivity $d \lambda_{B} / d \varepsilon=1.20 \mathrm{pm} / \mu \varepsilon$ at $1533.7 \mathrm{~nm}$ ) although some studies provide different values for a same kind of fiber: $p_{e}=0.28 \pm 0.02$ at 1536.1 in [34] or $p_{e}=0.205 \pm 0.004$ at $1533.7 \mathrm{~nm}$ in reference [36] (both for SMF28 fiber), our value for the SMF28e+ fiber at $1533.7 \mathrm{~nm}$ is $p_{e}=0.215 \pm 0.004$ corresponding to a strain sensitivity of $1.204 \mathrm{pm} / \mu \varepsilon$ at $1533.7 \mathrm{~nm}$. The origin of these differences can be either the type of grating or the nonlinear behavior of silica [25] in the large strain ranges used in other experiments.

The reflected wavelengths of the gratings measured during fabrication and the thermo-optic and strain-optic coefficients from Table 2 were introduced in equation (4) to obtain the phase index; the results are represented in Fig. 7, the plots are ordered from bottom (SMF28) to top (M5) according to the numerical aperture of the fibers. The index decreases with wavelength, index variations of the order of $10^{-4}$ can be clearly distinguished between two consecutive gratings in all data points. The SMF28 fiber has both the smallest index and index slope, while the erbium doped fiber presents the highest index and wavelength variation.

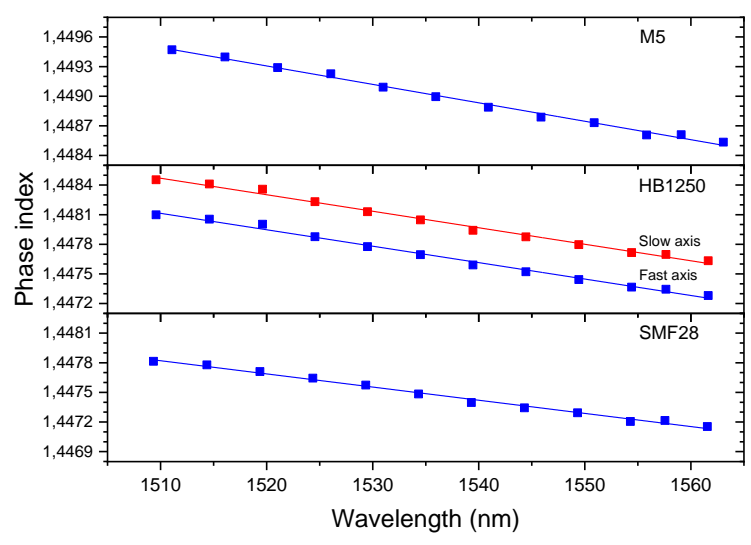

Fig. 7. Phase index as a function of the wavelength for the three fibers under test.

The parameters of the regression lines that fit the phase index and the correspondent values of the group indices are presented in Table 3. Fitting lines predict the phase index with an accuracy of $\pm 2 \times 10^{-5}$ for the SMF28 fiber and $\pm 3 \times 10^{-5}$ for the other two fibers, these figures covers all the contributions commented in section 2 and agrees reasonably well with the estimated overall error of Table 1 . The higher accuracy obtained for the telecommunications fiber is probably due its best axial uniformity [22]. For comparison, reference [18] reports a value of $1.44728 \pm 14 \times 10^{-5}$ for an unspecified type of Corning SMF28 fiber at $1534.06 \mathrm{~nm}$ and our result is $1.44750 \pm 2 \times 10^{-5}$ at the same wavelength, the difference between the two numbers is small and can be due either to the specific type of SMF28 fiber or to the larger error of the value presented in [18]. 
Table 3. Liner fit of the effective index as function of the wavelength and group index of the fibers.

\begin{tabular}{lcccccc}
\hline & \multicolumn{3}{c}{$n_{\text {eff }}=A+B(\lambda-1535)$} & & \multicolumn{2}{c}{ Group index. } \\
\cline { 2 - 3 } \cline { 6 - 7 } & $\mathrm{A}$ & $\mathrm{B}\left(\mathrm{nm}^{-1}\right)$ & Standard error & & Group index & Standard error \\
\hline SMF28 & 1,44749 & $-1.334 \times 10^{-5}$ & $\pm 2 \times 10^{-5}$ & & 1.4680 & \pm 0.0006 \\
M5 & 1.44903 & $-1.868 \times 10^{-5}$ & $\pm 3 \times 10^{-5}$ & & 1.4777 & \pm 0.0007 \\
HB1250 fast & 1.44770 & $-1.665 \times 10^{-5}$ & $\pm 3 \times 10^{-5}$ & & 1.4733 & \pm 0.0007 \\
HB1250 slow & 1.44805 & $-1.672 \times 10^{-5}$ & $\pm 3 \times 10^{-5}$ & & 1.4737 & \pm 0.0007 \\
\hline
\end{tabular}

The group index calculated from the index regression lines using equation (2). The group index varies between $1.4680 \pm 6 \times 10^{-4}$ for the telecommunication fiber and $1.4777 \pm 7 \times 10^{-4}$ for the erbium doped fiber, the slow and fast axes of the polarization maintaining fiber have a group index difference of 0.0004 . The group index uncertainty is determined by the error of the intercept of the regression lines, it is larger than phase index error. The Corning datasheet [37] reports a group index for the SMF 28e+ fiber of 1.4679 which is in excellent agreement with the value of 1.4680 we have obtained. Corning does not provide the uncertainty of the group index.

We have analyzed the residuals of the index data points obtained for all wavelengths and fibers. Fig. 8 (top) shows the deviation of data points from the fitting lines versus the phase masks used to write the gratings, the standard deviation of data points of the four sets of gratings is $2.30 \times 10^{-5}$. It can be observed in the top figure that each phase mask produces a systematic positive or negative deviation for all the fibers under test, the distribution of residuals grouped by fibers indicates that the incertitude of the phase mask pitch plays a significant role in the error of the experimental data. The difference of residuals between the different fibers has been calculated in order to quantify the phase mask contribution to the overall error of the phase index, the result is plotted in Fig. 8 (bottom). The standard error of the differences between fibers is $0.90 \times 10^{-5}$, since these differences account for errors other than the pitch error, we can estimate that the effect of the phase mask incertitude in the standard error of the experiment is about $\sqrt{2.30^{2}-0.90^{2}} \times 10^{-5}=2.1 \times 10^{-5}$, this contribution exceeds all other errors by a factor $7 / 3$. Thus, we can conclude that the precision of phase mask accuracy should be improved at least by a factor of five, hence a period accuracy better than $2 \mathrm{pm}$, to be considered as a secondary source of uncertainty for the determination of the phase index using this approach.

The intrinsic limit to the phase index accuracy is the fiber uniformity, which is comprised between $0.1 \times 10^{-5}$ and $1 \times 10^{-5}$ [22]. To achieve this level of error using fiber gratings a number of improvements should be carried out apart from improving the phase masks: temperature stability and precision of $0.1{ }^{\circ} \mathrm{C}$, gratings characterization with $1 \mathrm{pm}$ accuracy and gratings reflectivity about $0.5 \times 10^{-3}$. To achieve this goal, the fabrication rig should be thermally stabilized, a Burleigh wavelength meter could be used to characterize the gratings (or alternatively the setup presented in [32]), gratings with the required reflectivity can still be fabricated by the phase mask method and, finally, a phase mask with $2 \mathrm{pm}$ accuracy should be available.

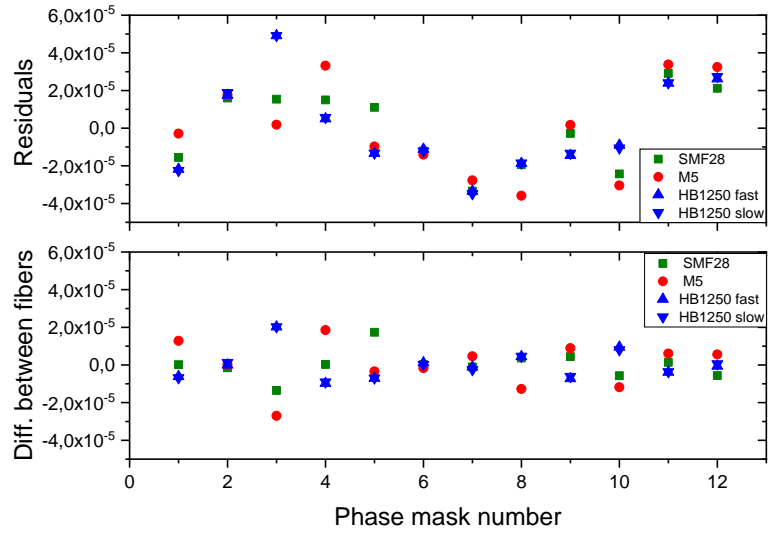

Fig. 8. Residuals of phase index fitting. Top: deviation of the phase index data points from the fitting lines for gratings fabricated with different phase masks. Bottom: differences of residuals between different fibers.

Finally, we present in Table 4 a comparison of some of our results with those previously reported in literature. We must point out that our measurement of the fiber phase index has a record accuracy of $2 \times 10^{-5}$; which is comparable to the value obtained by an interferometric technique for bulk materials in [8], our absolute value of the index in a SMF28 fiber is consistent with the result of reference [18]. We have measured group index with an accuracy of $6 \times 10^{-4}$, which is the same error that has been obtained by interferometry in references $[9,10]$, our value for a SMF28 fiber is in excellent agreement with the value provided by the fiber producer [37], unfortunately neither the error nor the measurement method is provided by Corning. Our values of the thermo-optic and strain optic coefficients agree well with the generic values proposed in [33], we found some discrepancy of values in the literature for gratings made in the same type of fiber, the precision we have obtained for these coefficients is not a record but it is among the best results. 
Table 4. Comparison of results of this work with previously reported data.

\begin{tabular}{|c|c|c|c|c|c|c|}
\hline Parameter & Value & $\begin{array}{l}\text { Standard } \\
\text { error }\end{array}$ & $\begin{array}{l}\text { Fiber or } \\
\text { material }\end{array}$ & $\begin{array}{l}\text { Wavelength } \\
\text { (nm) }\end{array}$ & Method & Reference \\
\hline Phase index & 1.44750 & $2 \times 10^{-5}$ & SMF $28 e+$ & 1534.06 & Gratings & [t.w.] ${ }^{(*)}$ \\
\hline Phase index & 1.44728 & $14 \times 10^{-5}$ & SMF 28 & 1534.06 & Gratings & [18] \\
\hline Refractive index & 1.504426 & $1.1 \times 10^{-5}$ & Soda glass & 1592 & Interferometer & [8] \\
\hline Group index & 1.4680 & 0.0006 & SMF $28 e+$ & $\mathrm{C}$ band & Gratings & [t.w. $]^{(*)}$ \\
\hline Group index & 1.4679 & n. s. $\left.{ }^{*}\right)$ & SMF $28 e^{+}$ & $\mathrm{C}$ band & n.s. $\left.{ }^{*}\right)$ & {$[37]$} \\
\hline Group index & 1.4393 & 0.0007 & $\left.\operatorname{PCF}{ }^{*}\right)$ & 632.82 & Interferometer & {$[9,10]$} \\
\hline$\zeta\left(\mathrm{K}^{-1}\right)$ & $6.03 \times 10^{-6}$ & $0.05 \times 10^{-6}$ & SMF $28 e^{+}$ & 1548.7 & Gratings & [t.w. $]^{(*)}$ \\
\hline$\zeta\left(\mathrm{K}^{-1}\right)$ & $6.16 \times 10^{-6}$ & n.s. $\left(^{*}\right)$ & n.s. ${ }^{(*)}$ & 1548.7 & Estimation & [33] \\
\hline$\zeta\left(\mathrm{K}^{-1}\right)$ & $5.32 \times 10^{-6}$ & n.s. $\left.{ }^{*}\right)$ & SMF 28 & 1548.7 & Gratings & {$[32]$} \\
\hline$\zeta\left(\mathrm{K}^{-1}\right)$ & $7.5 \times 10^{-6}$ & $0.3 \times 10^{-6}$ & SMF 28 & 1544.2 & Gratings & {$[34]$} \\
\hline$p_{e}$ & 0.215 & 0.004 & $S M F 28 e+$ & 1533.7 & Gratings & [t.w. $]^{(*)}$ \\
\hline$p_{e}$ & 0.22 & n.s. $\left(^{*}\right)$ & n.s. $\left.{ }^{*}\right)$ & 1533.7 & Estimation & {$[33]$} \\
\hline$p_{e}$ & 0.28 & 0.02 & SMF 28 & 1536.1 & Gratings & {$[34]$} \\
\hline$p_{e}$ & 0.205 & 0.004 & SMF 28 & 1533.7 & Gratings & [36] \\
\hline
\end{tabular}

\section{CONCLUSIONS}

We have measured the effective modal index of single-mode optical fibers as a function of the wavelength using weak fiber Bragg gratings. The method has been applied to a variety of fibers such as telecommunication, erbium doped and polarization maintaining; we have obtained similar results in terms of accuracy for all the kinds of fibers under test. The phase index has been obtained with an accuracy of $2 \times 10^{-5}$, which is a record that improves the previous value of $14 \times 10^{-}$ 5 reported in [18] using Bragg gratings and is close to the $1.1 \times 10^{-5}$ obtained by interferometry in bulk materials in reference [8]. It has been demonstrated the linear dependence of the phase index on the wavelength using gratings in the wavelength interval between 1505 and $1565 \mathrm{~nm}$. For the first time we have obtained the group index of optical fibers using Bragg gratings, the group refractive index of the fibers is calculated from the phase index width an accuracy of $7 \times 10^{-4}$, the phase index of the SMF28 fiber is in excellent agreement with the value provided by Corning. The main source of incertitude in the experiment is the accuracy of the period of the phase masks used to fabricate the gratings. All results are presented at zero strain and $22^{\circ} \mathrm{C}$ with the purpose of standardization. The strain-optic and thermo-optic coefficients of the fibers have been measured as a function of the wavelength and have been used to correct the refractive indices, the accuracy of both coefficients is better than $2 \%$. Our values of the coefficients are the closest to those of the pristine fiber reported so far because we have used the weakest gratings. The method can be extended to other types of fibers in which weak gratings can be inscribed, including few mode fibers, multiple core fibers, active fibers or large mode area fibers among others. Our results could be improved by using phase masks with an accuracy of $2 \mathrm{pm}$.

Funding: The authors acknowledge founding of the Ministerio de Ciencia e Innovación de España (project number PID2019-104276RBI00) and the Generalitat Valenciana of Spain (ref.: PROMETEO-2019048).
Disclosures: The authors declare no conflicts of interest.

\section{REFERENCES}

1. M. Young, "Optical fiber index profiles by the refracted -ray method (refracted near field scanning)," Appl. Opt. 20, 3415-3421 (1981).

2. S. Firstov, A. Khegai, K. Riumkin, Y. Ososkov, E. Firstova, M. Melkunov, S. Alyshev, E. Evlampieva, L. Iskhakova, A. Lobanov, V. Khopin, A. Abramov, M. Yashkov, and A. Guryanov, "Bend-insensitive bismuth-doped P2O5$\mathrm{SiO} 2$ glass core fiber for a compact O-band amplifier," Opt. Lett. 45, 25762579 (2020).

3. B. L. Bachim, T. K. Gaylord, and S. C. Mettler, "Refractive index profiling of azimutally asymmetric optical fibers by microinterferometric optical phase tomography," Opt. Lett. 30, 1126-1128 (2005).

4. A. Roberts, E. Ampem-Lassen, A. Barty, K. A. Nugent, G. W.Baxter, N. M. Dragomir, and S. T. Huntington, "Refractive-index profiling of optical fibers with axial symmetry by use of quantitative phase microscopy," Opt. Lett. 27, 2061 (2002).

5. https://cargille.com/wp-content/uploads/2018/07/Fused-Silica-MatchingLiquid-Code-06350.pdf

6. D. Derickson editor, "Fiber optic test and measurement," (Prentice HallI PTR, 1998).

7. A. C. P. Rocha, J. R. Silva, S. M. Lima, L. A. O. Nunes, and L. H. C. Andrade, "Measurements of refractive indices and thermo-optical coefficients using a white-light Michelson interferometer," Appl. Opt. 55, 6639-6642 (2016).

8. H.J. Choi, H.H. Lim, H. S. Moon, T.B. Eom, J.J. Ju, and M. Cha, "Measurement of refractive index and thickness of transparent plate by dual-wavelength interference," Opt. Express 18, 9429-9434 (2010).

9. J. Vengelis, V. Jarutis, and V. Sirutkaitis, "Measurement of the phase refractive index of a photonic crystal fiber mode," Opt. Lett. 43, 25712574 (2018).

10. O. Feinberg, J. Drori, and Y. Rosenberg, "Comment on "Measurement of the phase refractive index of a photonic crystal fiber mode"," Opt. Lett. 44, 85 (2019). 
11. X. Xu, Z. Zhang, H. Zhan, H. Zhao, W. Xia, M. He, J.Li, J. Zhai and H. Wu, "Long distance measurement by dynamic optical frequency comb," Opt. Express 28, 4398-4411 (2020).

12. K. Wada, S. Matsukura, A. Tanaka, T. Matsuyama, and H. Horinaka, "Precise measurement of single-mode fiber lengths using a gain-switched distributed feedback laser with delayed optical feedback," Opt. Express 23, 23013-23019 (2015)

13. C. Schulze, J. Wilde, R. Brüning, S. Schröter, and M. Duparré, "Measurement of effective refractive index differences in multimode optical fibers based on modal decomposition," Opt. Lett. 39, 5810-5813 (2014).

14. J. M. Savolainen, L. Grüner-Nielsen, P. Kristensen, and P. Balling, "Measurement of refractive-index differences in a few-mode fiber by axial fiber stretching," Opt. Express 20, 18646-18651 (2012).

15. E.P. Alcusa-Saez, A. Díez, M. Gonzalez-Herráez, and M.V. Andrés, "Improved time-resolved acousto-optic technique for optical fiber analysis of axial non-uniformities by using edge interrogation," Opt. Express 23, 7345-7349 (2015).

16. C. S. Kim, Y. G. Han, R. M. Sova, U. C. Paek, Y. Chung, and J. U. Kang, "Optical fiber modal birefringence measurement based on Lyot-Sagnac interferometer," IEEE Photon. Technol. Lett. 15, 269-271 (2003).

17. L. Costa, R. Magalhaes, L. Palmieri, H. Martins, S. Martínez-López, M. R. fernández-Ruiz, and M. González-Herraez, "Fast and direct measurement of the linear birefringence profile in standard single-mode optical fibers," Opt. Lett. 45, 623-626 (2020).

18. F. Jülich, and J. Roths, "Determination of the effective refractive index of various single ode fibers for fiber Bragg grating sensors applications," in Proceedings Sensors+Test Conference (2009), pp. 119-124.

19. R. Kashyap "Fiber Bragg gratings," (Academic Press, 1999).

20. G. M. Noah, Y. Bao, and T. K. Gaylord, "Cross sectional refractive-index variations in fiber Bragg gratings measured by quantitative phase imagin," Opt. Lett. 45, 53-56 (2020).

21. D. Marcuse,"Loss analysis in single-mode fiber splices," The Bell System Technical Journal 56, 703-718 (1977).

22. S. Loranger, and R. Kashyap, "Are optical fibers really uniform? Measurement of refractive index on a centimeter scale," Opt. Lett. 42, 1832-1835 (2017).

23. L. Poveda-Wong, J. L. Cruz, M. Delgado-Pinar, X. Roselló-Mechó, A. Díez and M.V. Andrés, "Fabrication of long period fiber gratings of subnanometric bandwidth," Opt. Let. 42, 1265-1268 (2017).

24. https://www.heraeus.com/en/hca/fused silica quartz knowledge base 1/properties 1/properties hca.htm

25. M. Guerette, C.R.Kurkjjian, S. Semjonov, and L. Huang, "Nonlinear elasticity of silica glass," J. Am. Ceram. Soc. 99, 841-848 (2016).

26. https://www.ign.es/web/ign/portal/grv-gravedad-absoluta

27. G. Pan, N. Yu, B. Meehan, T. W. Hawkins, J. Ballato, and P.D. Dragic, "Thermo-optic coefficient of $\mathrm{B}_{2} \mathrm{O}_{3}$ and $\mathrm{GeO}_{2}$ co-doped fibers," Optical Materials Express, 10, 1509-1521 (2020).

28. P.D. Dragic, J. Ballato, S. Morris, and T. W. Hawkins, "Pockels' coefficients of alumina and aluminosilicate optical fiber," J. Opt. Soc. Am. B 30, 244250 (2013).

29. T. N. Vasudevan, and R.S. Krishnan, "Dispersion of the stress-optic coefficient in glasses," J. Phys. D: Appl. Phys. 5, 2383-2387 (1972).

30. G. Ghosh, "Temperature dispersion of refractive indexes in some silicate fiber glasses," IEEE Photon. Technol. Lett. 6, 431-433 (1994).

31. G. Adamovsky, S. F. Lyuksyutov, J. R. Mackey, B. M. Floyd, U. Abeywikrema, I. Fedin, and M. Rackaitis, "Peculiarities of thermo-optic coefficient under different temperature regimes in optical fibers containing Bragg gratings," Opt. Communications 285, 766-773 (2012).

32. G. M. H. Flockhart, R. R. J. Maier, J. S. Barton, W. N. MacPherson, J. D. C. Jones, K. E. Chisholm, L. Zhang, and I. Bennion, "Quadratic behavior of fiber Bragg grating temperature coefficients," Appl. Opt. 43, 2744-2751 (2004).
33. A.D. Kersey, M. A. Davis, H.J. Patrick, M- LeBlanc, K.P. Koo, C.G. Askins, M.A. Putnam, and E.J. Friebele, "Fiber grating sensors," J. Lightwave Technol. 15, 1442-1461 (1997).

34. T. Osuch, A. Anuszkiewicz, K. Markowski, A. Filiplowski, D. Pysz, R. Kasztelanic, R. Stepien, M. Klimczak, and R. Buczynski, "Inscription of Bragg gratings in nanostructured graded index single-mode fibers" Opt. Express 27, 13721- 13732 (2019).

35. S Pal, T. Sun, K.T.V. Grattan, S. A. Wade, S. F. Collins, G. W. Baxter, B. Dussardier, and G. Monnom, "Non-linear temperature dependence of Bragg gratings written in different fibres, optimised for sensor applications over a wide range of temperatures," Sensors and Actuators A 112, 211219 (2004)

36. J. Roths, and F. Jülich, "Determination of strain sensitivity of free fibre Bragg gratings," in Proc. SPIE 7003, 700308, (2008).

37. https://www.corning.com/cala/es/products/communicationnetworks/products/fiber/smf-28e-.html 